\section{EXCESSO DE PESO EM ADOLESCENTES: PAPEL MODERADOR DO SEXO E DA ESCOLARIDADE MATERNA}

\author{
Overweight in adolescents: moderating role of gender and \\ mother's education level
}
Exceso de peso en adolescentes: papel moderador del sexo y de la escolaridad materna

\section{RESUMO}

Objetivo: Verificar o efeito moderador do sexo e da escolaridade materna na relação entre excesso de peso e fatores associados em adolescentes. Métodos: Estudo transversal descritivo, realizado com 945 escolares, de ambos os sexos, entre 14 e 18 anos, de Ponta Grossa, Paraná. Foram coletadas as medidas de peso e estatura. Os adolescentes preencheram um questionário sobre dados sociodemográficos (sexo, idade, ocupação/trabalho, moradia do adolescente e escolaridade do pai e da mãe), comportamento sedentário (tempo assistindo TV/computador), participação nas aulas de Educação Física e prática de atividade física. Para análise dos dados foi utilizada a regressão de Poisson no programa STATA 9.2, adotandose um nível de significância de $p<0,05$. Resultados: A prevalência de excesso de peso foi de $18,8 \%$. Houve associação entre excesso de peso e as variáveis idade e comportamento sedentário. Porém, quando as estimativas foram ajustadas para todas as outras variáveis independentes, apenas as idades de 15 e 16 anos mantiveram-se associadas ao excesso de peso. Foi observado um efeito moderador do sexo na associação entre excesso de peso e comportamento sedentário, e um efeito moderador da escolaridade materna na associação entre idade e prevalência de excesso de peso dos adolescentes. Conclusão: A prevalência de excesso de peso entre os adolescentes foi menor na faixa dos 14 aos 16 anos. No entanto, o aumento da idade e a presença de comportamentos sedentários foram associados ao menor risco de excesso de peso entre os meninos cujas mães possuem menor escolaridade. No caso das meninas, um menor risco de excesso de peso foi observado quando as mães apresentavam maior escolaridade.

Descritores: Adolescente; Sobrepeso; Obesidade.

\section{ABSTRACT}

Objective: To investigate the moderating effect of gender and mother's education level on the relation between overweight and associated factors in adolescents. Methods: This is a descriptive cross-sectional study, performed with 945 students, of both sexes, aged between 14 and 18 years, of Ponta Grossa, Paraná. Weight and height measurements were collected. The adolescents filled out a questionnaire addressing sociodemographic information (gender, age, occupation/work, adolescent's address and parents' level of education), sedentary behavior (time watching TV/at the computer), participation in Physical Education classes and practice of physical activities. For data analysis, Poisson regression was used in STATA 9.2 software, adopting a significance level of $p<0.05$. Results: The prevalence of overweight among the adolescents was $18.8 \%$. There was association between overweight and the variables age and sedentary behavior. However, when the estimates were adjusted for all the other independent variables, only the ages of 15 and 16 remained associated with overweight. A moderating effect of gender was observed in the association between overweight and sedentary behavior, and a moderating effect of mother's education on the association between adolescents' age and prevalence of overweight. Conclusion: The prevalence of overweight in adolescents was lower in the age range of 14 to 16 years old. Nevertheless, the age increase and the presence of sedentary behaviors were associated to lower risk of being overweight among boys whose mothers had lower formal schooling. As for the girls, a lower risk of overweight was observed when mothers presented higher level of education.

Descriptors: Adolescent; Overweight; Obesity.
Artigo Original
1) Universidade Federal do Paraná - UFPR Curitiba (PR) - Brasil

2) Universidade Federal de Santa Catarina UFSC - Florianópolis (SC) - Brasil

3) Pontifícia Universidade Católica do Paraná - PUC - Curitiba (PR) - Brasil
Recebido em: 03/03/2016 Revisado em: 10/05/2016 Aceito em: 12/09/2016 


\section{RESUMEN}

Objetivo: Verificar el efecto moderador del sexo y de la escolaridad materna en la relación entre el exceso de peso y factores asociados en adolescentes. Métodos: Estudio transversal descriptivo realizado con 945 escolares de ambos los sexos entre los 14 y 18 años, de Ponta Grossa, Paraná. Fueron recogidas las medidas de peso y altura. Los adolescentes rellenaron a un cuestionario sobre sus datos sociodemográficos (sexo, edad, ocupación/trabajo, vivienda del adolescente y escolaridad del padre y de la madre), el comportamiento sedentario (tiempo mirando la TV/ordenador), la participación en las clases de Educación Física y la práctica de actividad física. Para el análisis de datos se utilizó la regresión de Poisson en el programa STATA 9.2, adoptándose un nivel de significación de $p<0,05$. Resultados: La prevalencia del exceso de peso fue del 18,8\%. Hubo asociación entre el exceso de peso y las variables edad y conducta sedentaria. Sin embargo, cuando las estimativas fueron ajustadas para todas las otras variables independientes solamente las edades entre 15 y 16 años se mantuvieron asociadas con el exceso de peso. Se observó un efecto moderador del sexo para la asociación entre el exceso de peso y la conducta sedentaria y un efecto moderador de la escolaridad materna para la asociación entre la edad y la prevalencia de exceso de peso de los adolescentes. Conclusión: La prevalencia de exceso de peso entre los adolescentes fue menor en la franja de edad entre los 14 y 16 años. Sin embargo, el aumento de la edad y la presencia de conductas sedentarias estuvieron asociados al menor riesgo de exceso de peso entre los niños cuyas las madres tienen baja escolaridad. Para las niñas el menor riesgo de exceso de peso se observó cuando las madres presentaron mayor escolaridad.

Descriptores: Adolescente; Sobrepeso; Obesidad.

\section{INTRODUÇÃO}

O excesso de peso (EP) na adolescência está associado com obesidade na vida adulta e representa um importante fator de risco para doenças crônicas não transmissíveis ${ }^{(1-3)}$. No Brasil, a Pesquisa de Orçamentos Familiares (POF) 2008-2009, realizada pelo IBGE, destacou a prevalência de EP de 20,5\% nessa faixa etária, sendo 21,7\% nos meninos e $19,4 \%$ nas meninas ${ }^{(4)}$. Em 1989, essa prevalência era de 7,7\% nos meninos e 13,9\% nas meninas, demonstrando um acréscimo significativo ${ }^{(4)}$. A literatura aponta que fatores genéticos, sociodemográficos, comportamentais e ambientais estão associados com prevalência de EP em adolescentes $^{(5,6)}$.

A prevalência de EP é maior nos meninos ${ }^{(7,8)}$, em indivíduos com elevada condição socioeconômica ${ }^{(8,9)}$ e em escolares filhos de mães com maior escolaridade ${ }^{(9)}$. Deste modo, identificar os fatores associados ao EP é importante para identificar grupos com maior risco e propor intervenções mais específicas.
Apesar de existir razoável conhecimento sobre a prevalência e alguns fatores associados ao EP no Brasil, os estudos $^{(10,11)}$ têm enfatizado a relação estatística de modo independente entre as variáveis, ou seja, isola-se um fator e estima-se a sua relação com o EP. Portanto, não se investiga qual a interação entre duas ou mais variáveis.

Um estudo realizado com adolescentes na Alemanha identificou que a associação entre escolaridade dos pais e a prevalência de EP foi moderada pelo sexo. No entanto, esse efeito foi observado, apenas, no sexo feminino, ou seja, as filhas de pais com menor escolaridade possuíam maior prevalência de $\mathrm{EP}^{(12)}$. Em outro estudo, conduzido com adolescentes franceses ${ }^{(13)}$, os investigadores observaram que a idade foi negativamente associada com o EP no sexo feminino. Entre os meninos, a associação, apesar de não significativa, foi no sentido contrário. Os autores também identificaram que maior tempo assistindo televisão (TV) $(>2 \mathrm{~h} / \mathrm{dia})$ foi associado com maior prevalência de EP apenas entre as meninas.

Assim, fica evidente que apenas a análise da associação direta entre as variáveis pode não ser suficiente para um completo entendimento dos fatores associados ao EP. Deste modo, o presente estudo tem como objetivo verificar o efeito moderador do sexo e da escolaridade materna na relação entre excesso de peso e fatores associados em adolescentes.

\section{MÉTODOS}

Realizou-se um estudo transversal e descritivo, de base escolar ( $\mathrm{N}=6.597$ alunos) na rede pública de ensino do município de Ponta Grossa, Paraná, Brasil. Participaram do estudo indivíduos de ambos os sexos, matriculados nas séries do ensino médio, no período diurno, em escolas públicas da área urbana.

A amostragem foi estratificada de forma proporcional por conglomerados em dois estágios. Inicialmente, todas as escolas foram listadas e agrupadas de acordo com sua localização geográfica (norte, sul, leste, oeste e centro). Nove escolas foram sorteadas como unidades primárias de amostragem, adotando-se a estratificação pela região geográfica, o que garantiu a representatividade das regiões da cidade. O número de turmas selecionadas em cada escola foi definido de modo a alcançar a representatividade proporcional da área geográfica em relação ao município como um todo e a seleção ocorreu aleatoriamente.

Para o cálculo do tamanho da amostra, levou-se em consideração a prevalência de excesso de peso de $20 \%$ em adolescentes brasileiros ${ }^{(4)}$, intervalo de confiança de $95 \%$, erro de 3,0 pontos percentuais e efeito de delineamento de 1,5. A amostra mínima foi estimada em 928 indivíduos, sendo considerado elegível para o estudo o adolescente que se encontrava em sala de aula no dia da coleta e cujo 
responsável havia autorizado a sua participação. Adotou-se como critério de exclusão idade $\geq 19$ anos e adolescentes grávidas. No total, participaram do estudo 945 adolescentes.

A coleta de dados foi efetuada no período de março a dezembro de 2014 e ocorreu durante as aulas de Educação Física. Inicialmente, os pesquisadores entregaram o Termo de Consentimento Livre e Esclarecido (TCLE) para o responsável autorizar a participação do aluno no estudo. No dia seguinte, os adolescentes que desejavam participar do estudo preencheram o questionário orientado pelos pesquisadores. Posteriormente, os participantes foram para outra sala, onde foram aferidos o peso corporal e a estatura de acordo com os procedimentos da Organização Mundial da Saúde ${ }^{(14)}$. Foi utilizada uma balança digital, marca Plenna ${ }^{\circledR}$, com resolução de 100 gramas e capacidade máxima de $150 \mathrm{~kg}$, e um estadiômetro de parede, modelo Wiso ${ }^{\circledR}$, com altura máxima de $210 \mathrm{~cm}$ e resolução de 1 $\mathrm{cm}$. As coletas foram realizadas por dois pesquisadores treinados para a realização das medidas, com erro técnico de medida $<1 \%$.

A partir das medidas de peso e estatura, calculou-se o índice de massa corporal (IMC). Para classificação do estado nutricional, adotou-se a tabela normativa proposta para adolescentes brasileiros que considera sexo e idade ${ }^{(15)}$. Para análise, as categorias foram agrupadas em: não excesso de peso (baixo peso ou peso normal) e excesso de peso (sobrepeso ou obesidade).

O questionário aplicado aos alunos continha as variáveis independentes: sexo, idade (14 a 18 anos de idade), ocupação/trabalho (sim versus não), moradia do adolescente (mora com o pai ou mãe, mora com pai e com a mãe e outros) e escolaridade do pai e da mãe, a qual foi obtida por autorrelato dos adolescentes. Para tanto, os escolares relataram até que ano (série) o pai e a mãe estudaram. Posteriormente, os anos de estudo foram classificados em uma variável dicotômica: $<8$ anos de estudo (até ensino fundamental incompleto) e $\geq 8$ anos de estudo (ensino fundamental completo ou mais).

Ainda como variável independente, o comportamento sedentário foi avaliado por meio do tempo despendido em frente ao computador/TV por dia (de zero a oito ou mais horas), participação nas aulas de Educação Física (sim versus não) e prática de atividade física por meio do International Physical Activity Questionnaire (IPAQ) versão curta ${ }^{(16)}$. Para classificação do nível de atividade física, foram aplicadas recomendações específicas para adolescentes, sendo considerados inativos/insuficientemente ativos quem realizasse 0 a $299 \mathrm{~min} / \mathrm{sem}$ de atividades moderadas a vigorosas e ativos quem atingisse a recomendação de 300 $\mathrm{min} / \mathrm{sem}^{(17)}$.
Para a análise dos dados, utilizou-se inicialmente a estatística descritiva (distribuição de frequência absoluta e relativa), estratificada por sexo, para caracterização da amostra. Posteriormente, realizou-se a análise de regressão de Poisson bruta, e, em seguida, com ajuste para todas as variáveis por meio do programa STATA 9.2, adotando-se um nível de significância de $\mathrm{p}<0,05$. Para testar o efeito moderador do sexo e da escolaridade da mãe, foi criado um termo de interação destas variáveis (sexo e escolaridade da mãe) com todas as outras variáveis independentes. Esse modelo foi ajustado para todas as outras variáveis. Todas as análises consideraram o desenho amostral complexo por meio do comando svy do STATA.

O estudo foi aprovado pelo Comitê de Ética em Pesquisa com Seres Humanos da Universidade Estadual de Ponta Grossa (protocolo $\mathrm{n}^{\circ} .40 / 07$ ), de acordo com a Resolução no. 466/12 do Conselho Nacional de Saúde.

\section{RESULTADOS}

A amostra para análise foi de 945 adolescentes $(58,6 \%$ meninas, $\mathrm{n}=554)$, com idades entre 15 e 17 anos $(85,9 \%$, $\mathrm{n}=812)$, sem ocupação $(82 \%, \mathrm{n}=775)$, residentes com pai e mãe $(73,5 \%, n=695)$ e cujos pais possuem oito anos de estudo pelo menos (pais $41,1 \%, \mathrm{n}=388$; mães $40,2 \%, \mathrm{n}=380$ ). Cerca de $64,5 \%(n=609)$ dos adolescentes permanecem em frente à TV/computador por duas ou mais horas por dia, e a maior parte foi considerada ativa (meninos $76,5 \%$, $\mathrm{n}=299$; meninas $70,4 \%, \mathrm{n}=390$ ). A participação nas aulas de Educação Física foi de 88,3\% (n=834), sendo maior entre os meninos $(96,4 \%, \mathrm{n}=377$ versus $82,5 \%, \mathrm{n}=457 ; \mathrm{p}<0,01)$. A prevalência de excesso de peso foi de $18,8 \%(n=178$; $\left.\mathrm{IC}_{95 \%} 15,7-22,4\right)$, sendo mais elevada nos meninos $(21,7 \%$, $\mathrm{n}=85$ ). Outras características da amostra são apresentadas na tabela I.

Os adolescentes mais jovens apresentaram maior prevalência de excesso de peso $(39,4 \%$ aos 14 anos) seguido por uma diminuição até a idade de 16 anos $(14,6 \%)$ e posteriormente um novo aumento do excesso de peso nas idades de 17 e 18 anos (18,2\% e 20\%). Houve associação entre excesso de peso e as variáveis idade e comportamento sedentário (tempo assistindo TV/computador). Porém, quando as estimativas foram ajustadas para todas as outras variáveis independentes, apenas as idades de 15 e 16 anos mantiveram-se associadas com o excesso de peso (Tabela II).

Quando a análise foi realizada de forma estratificada por sexo (Tabela III), observou-se que o comportamento sedentário foi associado ao excesso de peso. Entre os meninos, quanto maior o tempo em frente TV/computador, menor a prevalência de excesso de peso ( $p$ de tendência $=0,013$ ), 
Tabela I - Características dos adolescentes do ensino médio de acordo com sexo (n=945). Ponta Grossa, PR, Brasil, 2014.

\begin{tabular}{|c|c|c|c|c|c|c|c|}
\hline \multirow[t]{2}{*}{ Variável } & \multicolumn{2}{|c|}{ Total } & \multicolumn{2}{|c|}{$\begin{array}{c}\text { Masculino } \\
(\mathrm{n}=\mathbf{3 9 1}, \mathbf{4 1 , 4 \% )}\end{array}$} & \multicolumn{2}{|c|}{$\begin{array}{c}\text { Feminino } \\
(\mathrm{n}=554, \mathbf{5 8 , 6} \%)\end{array}$} & \multirow[t]{2}{*}{ p-valor } \\
\hline & $\mathbf{n}$ & $\%$ & n & $\%$ & $n$ & $\%$ & \\
\hline \multicolumn{8}{|l|}{ Idade } \\
\hline 14 & 33 & 3,5 & 13 & 3,3 & 20 & 3,6 & 0,98 \\
\hline 15 & 263 & 27,8 & 109 & 27,9 & 154 & 27,8 & \\
\hline 16 & 280 & 29,6 & 119 & 30,4 & 161 & 29,1 & \\
\hline 17 & 269 & 28,5 & 111 & 28,4 & 158 & 28,5 & \\
\hline 18 & 100 & 10,6 & 39 & 10,0 & 61 & 11,0 & \\
\hline \multicolumn{8}{|l|}{ Série } \\
\hline $1^{\mathrm{a}}$ & 363 & 38,4 & 167 & 42,7 & 196 & 35,4 & 0,06 \\
\hline $2^{\mathrm{a}}$ & 292 & 30,9 & 116 & 29,7 & 176 & 31,8 & \\
\hline $3^{\mathrm{a}}$ & 290 & 30,7 & 108 & 27,6 & 182 & 32,9 & \\
\hline \multicolumn{8}{|l|}{ Ocupação/Trabalho } \\
\hline $\operatorname{Sim}$ & 170 & 18,0 & 97 & 24,8 & 73 & 13,2 & $<0,01$ \\
\hline Não & 775 & 82,0 & 294 & 75,2 & 481 & 86,8 & \\
\hline \multicolumn{8}{|l|}{ Moradia } \\
\hline Pai ou mãe & 174 & 18,4 & 73 & 18,7 & 101 & 18,2 & 0,56 \\
\hline Pai e Mãe & 695 & 73,5 & 291 & 74,4 & 404 & 72,9 & \\
\hline Outros & 76 & 8,0 & 27 & 6,9 & 49 & 8,8 & \\
\hline \multicolumn{8}{|l|}{ Escolaridade do pai } \\
\hline$<8$ anos & 557 & 58,9 & 221 & 56,5 & 336 & 60,7 & 0,20 \\
\hline$\geq 8$ anos & 388 & 41,1 & 170 & 43,5 & 218 & 39,4 & \\
\hline \multicolumn{8}{|l|}{ Escolaridade da mãe } \\
\hline$<8$ anos & 656 & 59,8 & 223 & 57,0 & 342 & 61,7 & 0,15 \\
\hline$\geq 8$ anos & 380 & 40,2 & 168 & 43,0 & 212 & 38,3 & \\
\hline \multicolumn{8}{|c|}{ (TV/computador) } \\
\hline$<1$ Hora & 101 & 10,7 & 35 & 9,0 & 66 & 11,9 & 0,45 \\
\hline 1-2 Horas & 235 & 24,9 & 97 & 24,8 & 138 & 24,9 & \\
\hline 3-4 Horas & 291 & 30,8 & 131 & 33,5 & 160 & 28,9 & \\
\hline 5-6 horas & 201 & 21,3 & 82 & 21,0 & 119 & 21,5 & \\
\hline 7-8 Horas & 117 & 12,4 & 46 & 11,8 & 71 & 12,8 & \\
\hline \multicolumn{8}{|l|}{ AFMV* } \\
\hline Inativo/Insuficientemente Ativo & 256 & 27,1 & 92 & 23,5 & 164 & 29,6 & 0,04 \\
\hline Ativo & 689 & 72,9 & 299 & 76,5 & 390 & 70,4 & \\
\hline \multicolumn{8}{|c|}{ Participação nas aulas de Educação Física } \\
\hline Não & 111 & 11,8 & 14 & 3,6 & 97 & 17,5 & $<0,01$ \\
\hline Sim & 834 & 88,3 & 377 & 96,4 & 457 & 82,5 & \\
\hline \multicolumn{8}{|l|}{ Estado nutricional } \\
\hline Normal & 767 & 81,2 & 306 & 78,3 & 461 & 83,2 & 0,06 \\
\hline Sobrepeso/Obesidade & 178 & 18,8 & 85 & 21,7 & 93 & 16,8 & \\
\hline
\end{tabular}

* Atividade Física moderada e vigorosa. Ativo: $\geq 300$ minutos/semana. 
Tabela II - Associação entre prevalência de excesso de peso e variáveis sociodemográficas, de saúde e comportamentais em adolescentes do ensino médio (n=945). Ponta Grossa, PR, Brasil, 2014.

\begin{tabular}{|c|c|c|c|c|c|c|c|c|}
\hline \multirow[b]{2}{*}{ Variável } & \multirow[b]{2}{*}{ Categoria } & \multirow{2}{*}{$\begin{array}{l}\text { Excesso de } \\
\operatorname{peso}(\%)\end{array}$} & \multicolumn{3}{|c|}{ Análise bruta } & \multicolumn{3}{|c|}{ Análise ajustada } \\
\hline & & & $\mathbf{R P}$ & IC95\% & p-valor & $\mathbf{R P}$ & IC95\% & p-valor \\
\hline \multirow{2}{*}{ Sexo } & Masculino & 21,74 & 1,00 & & & 1,00 & & \\
\hline & Feminino & 16,79 & 0,77 & $(0,57-1,04)$ & 0,08 & 0,79 & $(0,60-1,04)$ & 0,09 \\
\hline \multirow{5}{*}{ Idade } & 14 & 39,4 & 1,00 & & & 1,00 & & \\
\hline & 15 & 20,9 & 0,53 & $(0,31-0,91)$ & 0,03 & 0,53 & $(0,31-0,89)$ & 0,02 \\
\hline & 16 & 14,6 & 0,37 & $(0,19-0,73)$ & 0,01 & 0,37 & $(0,19-0,74)$ & 0,01 \\
\hline & 17 & 18,2 & 0,46 & $(0,23-0,95)$ & 0,04 & 0,46 & $(0,21-1,03)$ & 0,06 \\
\hline & 18 & 20,0 & 0,51 & $(0,22-1,17)$ & 0,10 & 0,52 & $(0,21-1,28)$ & 0,13 \\
\hline \multirow{2}{*}{ Ocupação/Trabalho } & Sim & 20,0 & 1,08 & $(0,74-1,56)$ & 0,70 & 1,01 & $(0,73-1,38)$ & 0,96 \\
\hline & Não & 18,6 & 1,00 & & & 1,00 & & \\
\hline \multirow{3}{*}{ Moradia } & Pai ou mãe & 20,69 & 1,00 & & & 1,00 & & \\
\hline & Pai e Mãe & 18,99 & 0,92 & $(0,70-1,20)$ & 0,48 & 0,90 & $(0,69-1,18)$ & 0,40 \\
\hline & Outros & 13,16 & 0,64 & $(0,38-1,05)$ & 0,07 & 0,63 & $(0,35-1,16)$ & 0,12 \\
\hline \multirow{2}{*}{ Escolaridade do pai } & $<8$ anos & 18,13 & 1,00 & & & 1,00 & & \\
\hline & $\geq 8$ anos & 19,85 & 1,09 & $(0,85-1,42)$ & 0,44 & 1,06 & $(0,78-1,46)$ & 0,67 \\
\hline \multirow{2}{*}{ Escolaridade da mãe } & $<8$ anos & 18,76 & 1,00 & & & 1,00 & & \\
\hline & $\geq 8$ anos & 18,95 & 1,01 & $(0,68-1,50)$ & 0,96 & 0,99 & $(0,65-1,51)$ & 0,96 \\
\hline \multirow{5}{*}{$\begin{array}{l}\text { Comportamento sedentário } \\
\text { (TV/computador) em tempo }\end{array}$} & $<1$ Hora & 23,76 & 1,00 & & & 1,00 & & \\
\hline & 1-2 Horas & 18,72 & 0,79 & $(0,51-1,21)$ & 0,24 & 0,77 & $(0,50-1,20)$ & 0,22 \\
\hline & 3-4 Horas & 17,53 & 0,74 & $(0,49-1,12)$ & 0,13 & 0,69 & $(0,44-1,08)$ & 0,09 \\
\hline & 5-6 horas & 17,41 & 0,73 & $(0,55-0,98)$ & 0,04 & 0,69 & $(0,46-1,03)$ & 0,07 \\
\hline & $>7$ horas & 20,5 & 0,86 & $(0,43-1,73)$ & 0,51 & 0,85 & $(0,39-1,86)$ & 0,65 \\
\hline \multirow{2}{*}{ AFMV* } & Inativo/IA & 16,80 & 1,00 & & & 1,00 & & \\
\hline & Ativo & 19,59 & 1,17 & $(0,87-1,56)$ & 0,26 & 1,20 & $(0,91-1,58)$ & 0,18 \\
\hline \multirow{2}{*}{$\begin{array}{l}\text { Participação em aulas de } \\
\text { Educação Física }\end{array}$} & Não & 15,3 & 1,00 & & & 1,00 & & \\
\hline & Sim & 19,3 & 1,26 & $(0,89-1,79)$ & 0,19 & 1,17 & $(0,86-1,59)$ & 0,27 \\
\hline
\end{tabular}

* Atividade Física moderada e vigorosa. Ativo: $\geq 300$ minutos/semana. IA: Insuficientemente ativo. RP: Razão de prevalência; IC95\%: Intervalo de confiança de $95 \%$.

porém, tal associação foi somente marginalmente associada entre as meninas ( $\mathrm{p}$ de tendência $=0,09)$. O efeito moderador do sexo foi significativo ( $p$ de tendência $=0,006$ ), confirmando as análises estratificadas e indicando que a associação entre o tempo de exposição em frente a TV/ computador e prevalência de excesso de peso é específica para cada um dos sexos.
Na tabela IV, observou-se que a associação entre sexo e excesso peso foi estatisticamente significativa $(p<0,05)$ para as adolescentes com mães com 8 anos ou mais de escolaridade, e que, dessa forma, apresentaram menor risco de excesso de peso. Por outro lado, o comportamento sedentário esteve associado com menor risco de excesso de peso somente entre os adolescentes cujas as mães 
Tabela III - Associação entre excesso de peso e variáveis sociodemográficas, de saúde e comportamentais estratificada por sexo e teste de interação em adolescentes do ensino médio. Ponta Grossa, PR, Brasil, 2014.

\begin{tabular}{|c|c|c|c|c|c|c|c|}
\hline \multirow{3}{*}{ Variável } & \multirow{3}{*}{ Categoria } & \multicolumn{4}{|c|}{ Sexo } & \multirow{2}{*}{\multicolumn{2}{|c|}{$\begin{array}{c}\text { Efeito moderador do } \\
\text { sexo }\end{array}$}} \\
\hline & & \multicolumn{2}{|c|}{ Masculino } & \multicolumn{2}{|c|}{ Feminino } & & \\
\hline & & RP & IC95\% & $\mathbf{R P}$ & IC95\% & RP & IC95\% \\
\hline \multirow{5}{*}{ Idade } & 14 & 1,00 & & 1,00 & & 1,00 & \\
\hline & 15 & 0,72 & $(0,27-1,90)$ & 0,41 & $(0,16-1,02)$ & 0,57 & $(0,11-2,83)$ \\
\hline & 16 & 0,42 & $(0,15-1,13)$ & 0,34 & $(0,11-1,06)$ & 0,82 & $(0,14-4,72)$ \\
\hline & 17 & 0,49 & $(0,24-1,02)$ & 0,44 & $(0,14-1,40)$ & 0,90 & $(0,21-3,91)$ \\
\hline & 18 & 0,67 & $(0,30-1,48)$ & 0,41 & $(0,12-1,38)$ & 0,61 & $(0,15-2,57)$ \\
\hline \multirow{2}{*}{ Ocupação/Trabalho } & Sim & 0,98 & $(0,65-1,47)$ & 1,06 & $(0,76-1,48)$ & 1,08 & $(0,60-1,97)$ \\
\hline & Não & 1,00 & & 1,00 & & 1,00 & \\
\hline \multirow{3}{*}{ Moradia } & Pai ou mãe & 1,00 & & 1,00 & & 1,00 & \\
\hline & Pai e Mãe & 1,03 & $(0,75-1,44)$ & 0,83 & $(0,54-1,25)$ & 0,80 & $(0,47-1,36)$ \\
\hline & Outros & 0,51 & $(0,13-1,97)$ & 0,72 & $(0,45-1,15)$ & 1,42 & $(0,34-6,04)$ \\
\hline \multirow{2}{*}{ Escolaridade do pai } & $<8$ anos & & & & & & \\
\hline & $\geq 8$ anos & 1,00 & $(0,77-1,31)$ & 1,16 & $(0,75-1,80)$ & 1,16 & $(0,70-1,92)$ \\
\hline \multirow{2}{*}{ Escolaridade da mãe } & $<8$ anos & 1,00 & & 1,00 & & 1,00 & \\
\hline & $\geq 8$ anos & 2,75 & $(0,90-8,39)$ & 0,81 & $(0,34-1,97)$ & 0,30 & $(0,07-1,22)$ \\
\hline \multirow{5}{*}{$\begin{array}{l}\text { Comportamento sedentário } \\
\text { (TV/computador) em tempo }\end{array}$} & $<1$ Hora & 1,00 & & 1,00 & & 1,00 & \\
\hline & 1-2 Horas & 0,72 & $(0,41-1,28)$ & 0,77 & $(0,24-2,48)$ & 1,06 & $(0,24-4,62)$ \\
\hline & 3-4 Horas & 0,44 & $(0,22-0,87)$ & 1,16 & $(0,55-2,41)$ & 2,63 & $(0,87-7,99)$ \\
\hline & 5-6 horas & 0,43 & $(0,20-0,93)$ & 1,16 & $(0,54-2,49)$ & 2,73 & $(0,70-10,61)$ \\
\hline & $>6$ Horas & 0,33 & $(0,12-0,92)$ & 1,67 & $(0,50-5,61)$ & 5,13 & $(0,96-27,43)$ \\
\hline \multirow{2}{*}{ AFMV* } & Inativo/IA & 1,00 & & 1,00 & & 1,00 & \\
\hline & Ativo & 1,23 & $(0,85-1,79)$ & 1,08 & $(0,69-1,69)$ & 0,88 & $(0,49-1,60)$ \\
\hline \multirow{2}{*}{$\begin{array}{l}\text { Participação nas aulas de } \\
\text { Educação Física }\end{array}$} & Não & 1,00 & & 1,00 & & 1,00 & \\
\hline & Sim & 3,12 & $(0,41-2,35)$ & 1,02 & $(0,70-1,49)$ & 0,33 & $(0,03-3,33)$ \\
\hline
\end{tabular}

*AFMV: Atividade Física moderada e vigorosa. Ativo: $\geq 300$ minutos/semana. IA: Insuficientemente ativo. RP: Razão de prevalência; IC95\%: Intervalo de confiança de 95\%.

possuem menor escolaridade ( $<8$ anos de estudo). Também se observou um efeito moderador positivo da escolaridade da mãe na associação entre idade e prevalência de excesso de peso nos adolescentes. Os resultados indicam que esta associação é significativamente diferente $(\mathrm{p}<0,05)$, sendo verdadeira apenas para os adolescentes cujas mães possuem menos de oito anos de escolaridade.

\section{DISCUSSÃO}

A prevalência de excesso de peso entre os adolescentes foi de 18,8\% ( $\left.\mathrm{IC}_{95 \%}: 15,7-22,4\right)$. Esses resultados são semelhantes (13 a 23\%) ao relatado entre adolescentes de outros países ${ }^{(18-20)}$ e da região sul do Brasil ${ }^{(21-23)}$. Um estudo de revisão sistemática para estimar a prevalência de sobrepeso e obesidade em crianças e adolescentes na América Latina encontrou que $16,6 \%$ a $35,8 \%$ dos adolescentes de 12 a 19 anos apresentam sobrepeso e obesidade ${ }^{(24)}$.

Houve maior prevalência de excesso de peso entre os meninos $(21,7 \%)$. Esses resultados corroboram com investigações nacionais ${ }^{(21,25)}$ e internacionais ${ }^{(7,26,27)}$. De maneira geral, meninas são menos satisfeitas com o peso corporal e sofrem maior pressão social para manter um padrão de "magreza", isso pode contribuir para um maior controle do peso corporal ${ }^{(28)}$.

Os adolescentes mais jovens apresentaram maior prevalência de excesso de peso ( $39,4 \%$ aos 14 anos), seguido de uma diminuição até os 16 anos $(14,6 \%)$ e posteriormente 
Tabela IV - Associação entre a prevalência de excesso de peso e variáveis sociodemográficas, de saúde e comportamentais estratificada por escolaridade da mãe e teste de interação em adolescentes do ensino médio. Ponta Grossa, PR, Brasil, 2014.

\begin{tabular}{|c|c|c|c|c|c|c|c|}
\hline \multirow{3}{*}{ Variável } & \multirow{3}{*}{ Categoria } & \multicolumn{4}{|c|}{ Escolaridade da mãe } & \multirow{2}{*}{\multicolumn{2}{|c|}{$\begin{array}{c}\text { Efeito moderador } \\
\text { da escolaridade da } \\
\text { mãe }\end{array}$}} \\
\hline & & \multicolumn{2}{|c|}{$<8$ anos } & \multicolumn{2}{|c|}{$\geq 8$ anos } & & \\
\hline & & RP & IC95\% & RP & IC95\% & $\mathbf{R P}$ & IC95\% \\
\hline \multirow{2}{*}{ Sexo } & Masculino & 1,00 & & 1,00 & & 1,00 & \\
\hline & Feminino & 0,99 & $(0,70-1,41)$ & 0,53 & $(0,30-0,97)$ & 0,54 & $(0,26-1,11)$ \\
\hline \multirow{5}{*}{ Idade } & 14 & 1,00 & & 1,00 & & 1,00 & \\
\hline & 15 & 0,45 & $(0,27-0,76)$ & 0,78 & $(0,34-1,79)$ & 1,72 & $(0,84-3,53)$ \\
\hline & 16 & 0,26 & $(0,14-0,48)$ & 0,74 & $(0,23-2,35)$ & 2,87 & $(1,06-7,81)$ \\
\hline & 17 & 0,36 & $(0,18-0,73)$ & 0,81 & $(0,35-1,86)$ & 2,26 & $(1,18-4,35)$ \\
\hline & 18 & 0,32 & $(0,11-0,87)$ & 1,11 & $(0,40-3,09)$ & 3,51 & $(1,43-8,62)$ \\
\hline \multirow{2}{*}{ Ocupação/Trabalho } & Sim & 1,30 & $(0,86-1,96)$ & 0,77 & $(0,30-1,96)$ & 1,69 & $(0,49-5,88)$ \\
\hline & Não & 1,00 & & 1,00 & & 1,00 & \\
\hline \multirow{3}{*}{ Moradia } & Pai ou mãe & 1,00 & & 1,00 & & 1,00 & \\
\hline & Pai e Mãe & 0,85 & $(0,60-1,21)$ & 1,01 & $(0,65-1,58)$ & 1,19 & $(0,66-2,12)$ \\
\hline & Outros & 0,53 & $(0,23-1,23)$ & 0,84 & $(0,31-2,29)$ & 1,58 & $(0,34-7,35)$ \\
\hline \multirow{2}{*}{ Escolaridade do pai } & $<8$ anos & 1,00 & & 1,00 & & 1,00 & \\
\hline & $\geq 8$ anos & 0,98 & $(0,64-1,50)$ & 1,31 & $(0,91-1,87)$ & 1,34 & $(0,81-2,22)$ \\
\hline \multirow{5}{*}{$\begin{array}{l}\text { Comportamento sedentário } \\
\text { (TV/computador) em tempo }\end{array}$} & $<1$ Hora & 1,00 & & 1,00 & & 1,00 & \\
\hline & 1-2 Horas & 0,62 & $(0,41-0,95)$ & 1,18 & $(0,53-2,64)$ & 1,90 & $(0,88-4,09)$ \\
\hline & 3-4 Horas & 0,78 & $(0,48-1,25)$ & 0,72 & $(0,38-1,36)$ & 0,93 & $(0,44-1,94)$ \\
\hline & 5-6 horas & 0,62 & $(0,42-0,90)$ & 0,98 & $(0,51-1,89)$ & 1,59 & $(0,79-3,22)$ \\
\hline & 7 horas ou mais & 0,84 & $(0,32-2,21)$ & 0,98 & $(0,43-2,21)$ & 1,17 & $(0,41-3,32)$ \\
\hline \multirow{2}{*}{ AFMV* } & Inativo/IA & 1,00 & & 1,00 & & 1,00 & \\
\hline & Ativo & 1,21 & $(0,81-1,81)$ & 1,11 & $(0,67-1,82)$ & 0,91 & $(0,47-1,76)$ \\
\hline Participação nas aulas de & Não & 1,00 & & 1,00 & & 1,00 & \\
\hline Educação Física & Sim & 0,93 & $(0,73-1,19)$ & 2,08 & $(0,62-6,97)$ & 2,23 & $(0,62-8,01)$ \\
\hline
\end{tabular}

*Atividade Física moderada e vigorosa. Ativo: $\geq 300$ minutos/semana. IA: Insuficientemente ativo. RP: Razão de prevalência; IC95\%: Intervalo de confiança de $95 \%$.

um novo aumento aos 17 e 18 anos (18,2\% para $20 \%)$. Esse resultado pode ser atribuído, em parte, à menor preocupação com a aparência física e, portanto, com o excesso de peso em adolescentes com menor idade. Assim, adolescentes mais jovens tendem a se preocupar menos com a saúde e com os padrões estéticos de beleza, o que pode favorecer o acúmulo de peso corporal nesta fase. No entanto, o aumento do excesso de peso em escolares mais velhos (17 a 18 anos) pode ser decorrente da diminuição na atividade física e o aumento do comportamento sedentário, muito comum nesta fase, devido ao aumento dos compromissos escolares (entrada na universidade), trabalho de meio período e consequentemente menor cuidado com hábitos saudáveis na sua rotina ${ }^{(29,30)}$.
$\mathrm{Na}$ análise estratificada por sexo, maior exposição a comportamentos sedentários foi associada negativamente com excesso de peso entre os meninos, porém, foi apenas marginalmente associado de maneira positiva com excesso de peso entre as meninas. Estes resultados indicam que a associação entre variáveis comportamentais, como o comportamento sedentário e o excesso de peso em adolescentes, pode ser específico para cada sexo. Padrão semelhante foi observado em uma amostra representativa de adolescentes australianos. Entre as adolescentes não foi observada associação entre exposição a comportamentos sedentários e o IMC, porém, a exposição a comportamentos sedentários foi inversamente associada ao $\mathrm{IMC}^{(31)}$. Ressaltase que, apesar do comportamento sedentário, os meninos 
participavam mais das aulas de Educação Física (quase $100 \%$ ) e também realizavam mais atividade física moderada e vigorosa. Então, apesar do comportamento sedentário, os meninos apresentam mais comportamentos ativos do que as meninas.

Dessa forma, as meninas parecem ser mais suscetíveis a desenvolver excesso de peso quando expostas a comportamentos sedentários em comparação com os meninos (associação marginal; $\mathrm{p}$ de tendência $=0,09$ ). Uma das hipóteses para explicar tal fato está relacionada aos menores níveis de atividade física entre as meninas ${ }^{(32)}$, ou seja, o menor gasto energético em meninas devese em parte a menor exposição à prática de atividades físicas moderadas e vigorosas. Assim, a exposição a comportamentos sedentários pode aumentar ainda mais a chance de desenvolver excesso de peso neste subgrupo.

Apesar de contraditório ao esperado, a associação inversa entre maior tempo em comportamentos sedentários e excesso de peso, observada no presente estudo, foi também observada em outras pesquisas ${ }^{(33)}$. Além disso, revisões sobre esta associação têm apresentado resultados inconsistentes ou efeito clinicamente irrelevante ${ }^{(34)}$. Tais inconsistências podem ser explicadas pelo efeito moderador de hábitos alimentares, principalmente durante alguns comportamentos sedentários, como assistir $\mathrm{TV}^{(35)}$. Ressalta-se também que comportamentos sedentários têm sido tratados como um comportamento distinto da atividade física, podendo estes coexistirem e afetarem de maneira independente indicadores de saúde como indicadores metabólicos e o excesso de peso.

Houve um efeito moderador da escolaridade materna na associação entre idade e prevalência de excesso de peso para os adolescentes cujas mães possuem menos de oito anos de estudo. Assim, os filhos de mães com menor escolaridade tendem a apresentar menor prevalência de excesso de peso em decorrência de uma condição socioeconômica menos favorecida e, portanto, menor poder de compra de produtos alimentícios. Estudos apontam que o alto nível de escolaridade materna corresponde a maior chance de sobrepeso e obesidade nos escolares ${ }^{(36,37)}$.

Destaca-se, no presente estudo, a abrangência da investigação e o tamanho da amostra, suficientes para garantir estimativas de prevalência com razoável precisão, além de um delineamento de base escolar, com seleção probabilística que contou com a medida objetiva de peso e estatura. Porém, existem limitações que devem ser consideradas, como a delimitação aos escolares do ensino médio da rede pública, que não permite extrapolar os resultados para todos os adolescentes da cidade. A cidade onde o estudo foi realizado é relativamente pequena (311.611 habitantes), e pode não representar cidades com características diferentes. $\mathrm{O}$ delineamento transversal também deve ser considerado, impossibilitando determinar o sentido causal das associações observadas. A utilização de questionário como forma de coleta de dados pode ser influenciada pelo viés de memória, principalmente em relação às medidas de atividade física e comportamento sedentário, porém, outros estudos utilizaram instrumentos similares e demonstraram resultados satisfatórios ${ }^{(38,39)}$. Por fim, a disponibilidade de medida de hábitos alimentares poderia ajudar no entendimento das inter-relações observadas no presente estudo, porém, tais dados não foram coletados e devem ser considerados na conclusão dos achados deste estudo.

Dessa forma, intervenções e futuros estudos com delineamento longitudinal devem ser realizados para que os resultados observados em estudos transversais possam ser confirmados, assim como verificar possíveis relações causais. Assim sendo, atenção especial deve ser dada aos comportamentos sedentários em meninas e adolescentes cujas mães possuem mais de oito anos de estudo. Ações devem ser direcionadas especificamente para estes grupos para a redução da prevalência de excesso de peso.

\section{CONCLUSÃO}

A prevalência de excesso de peso entre os adolescentes foi menor entre os 14 e 16 anos. No entanto, o aumento da idade e a presença de comportamentos sedentários foram associados ao menor risco de excesso de peso entre os meninos cujas mães possuem menor escolaridade. No caso das meninas, um menor risco de excesso de peso foi observado quando as mães apresentavam escolaridade maior ( $\geq 8$ anos de estudo).

\section{REFERÊNCIAS}

1. Lobstein T, Jackson-Leach R, Moodie ML, Hall KD, Gortmaker SL, Swinburn BA, et al. Child and adolescent obesity: part of a bigger picture. Lancet. 2015;385(9986):2510-20.

2. Callo G, Gigante DP, Barros FC, Horta BL. Lifetime overweight and obesity and body composition in adulthood: the 1982 Pelotas (Brazil) birth cohort study. Cad Saúde Pública. 2016;32(4):1-8.

3. Patton GC, Sawyer SM, Santelli JS, Ross DA, Afifi $\mathrm{R}$, Allen NB, et al. Our future: a Lancet commission on adolescent health and wellbeing. Lancet. 2016;387(10036):2423-78.

4. Instituto Brasileiro de Geografia e Estatística. Pesquisa de Orçamentos Familiares 208-2009. Antropometria e Estado Nutricional de crianças, adolescentes e adultos no Brasil [Internet]. Rio de Janeiro: IBGE. [acesso em 
2016 Set 23]. Disponível em: http://biblioteca.ibge. gov.br/visualizacao/livros/liv45419.pdf

5. Onis Md. Preventing childhood overweight and obesity. J Pediatr (Rio J). 2015;91(2):105-7.

6. Williams EP, Mesidor M, Winters K, Dubbert PM, Wyatt SB. Overweight and Obesity: Prevalence, Consequences, and Causes of a Growing Public Health Problem. Curr Obes Rep. 2015;4(3):363-70.

7. Tornquist L, Tornquist D, Reuter CP, Burgos LT, Burgos MS. Excess weight and high blood pressure in schoolchildren: prevalence and associated factors. J Human Growth Dev. 2015;25(2):216-23.

8. Souza GS, Quadros TMB, Gordia AP, Facina VB. Revisão de literatura sobre extremos antropométricos em crianças e adolescentes: prevalência, riscos à saúde e fatores sociodemográficos associados. Rev Aten Saúde. 2015;13(45):102-13.

9. Leal VS, Lira PIC, Oliveira JS, Menezes RC, Sequeira LAS, Arruda MA Neto, et al. Overweight in children and adolescents in Pernambuco State, Brazil: prevalence and determinants. Cad Saúde Pública. 2012;28(6):1175-82.

10. Mendes RC, Tavares CG, Figueredo GFD, Silva RL, Diniz KGD, Martins C, et al. Fatores associados ao excesso de peso e ao índice de massa corporal em três escolas de Itaúna-MG. Rev Med Minas Gerais. 2015;25(1):30-6.

11. D’Avila GL, Müller RL, Gonsalez PS, de Vasconcelos FdAG. Associação entre estado nutricional da mãe e a frequência, local e companhia durante as refeições com o sobrepeso/obesidade de adolescentes da cidade de Florianópolis, Brasil. Rev Bras Saude Matern Infant. 2015;15(3):289-99.

12. Plachta-Danielzik S, Landsberg B, Johannsen M, Lange D, Muller MJ. Determinants of the prevalence and incidence of overweight in children and adolescents. Public Health Nutr. 2010;13(11):1870-81.

13. Dupuy M, Godeau E, Vignes C, Ahluwalia N. Sociodemographic and lifestyle factors associated with overweight in a representative sample of 11-15 year olds in France: results from the WHO-Collaborative Health Behaviour in School-aged Children (HBSC) crosssectional study. BMC Public Health. 2011;11(442):111.

14. World Health Organization. Physical status: The use and interpretation of anthropometry [Internet]. Geneva: WHO. [acesso em 2016 Set 23]. Disponível em: http://apps.who.int/iris/bitstream/10665/37003/1/ WHO_TRS_854.pdf

15. Conde WL, Monteiro CA. Body mass index cutoff points for evaluation of nutritional status in Brazilian children and adolescents. J Pediatr (Rio J). 2006;82(4):266-72.

16. Guedes DP, Lopes CC, Guedes JERP. Reprodutibilidade e validade do Questionário Internacional de Atividade Física em adolescentes. Rev Bras Med Esporte. 2005;11(2):151-8.

17. Strong WB, Malina RM, Blimkie CJ, Daniels SR, Dishman RK, Gutin B, et al. Evidence based physical activity for school-age youth. J Pediatr. 2005;146(6):732-7.

18. Chorin E, Hassidim A, Hartal M, Havakuk O, Flint N, Ziv-Baran T, et al. Trends in adolescents obesity and the association between bmi and blood pressure: a cross-sectional study in 714,922 healthy teenagers. Am J Hypertens. 2015;28(9):1157-63.

19. Carroll MD, Navaneelan T, Bryan S, Ogden CL. Prevalence of obesity among children and adolescents in the United States and Canada [Internet]. Atlanta: Centers for Disease Control and Prevention; 2015. (NCHS Data Brief. Number 211) [acesso em 2016 Out 16]. Disponível em: https://www.cdc.gov/nchs/data/ databriefs/db211.pdf

20. Ogden CL, Carroll MD, Lawman HG, Fryar CD, Kruszon-Moran D, Kit BK, et al. Trends in Obesity Prevalence Among Children and Adolescents in the United States, 1988-1994 Through 2013-2014. JAMA. 2016;315(21):2292-9.

21. Cureau FV, Duarte PM, Santos DL, Reichert FF, Zanini RR. Overweight/obesity in adolescents from Santa Maria, Brazil: Prevalence and associated factors. Rev Bras Cineantropom Desempenho Hum. 2012;14(5):517-26.

22. Benedet J, Assis MAA, Calvo MCM, Andrade DF. Excesso de peso em adolescentes: explorando potenciais fatores de risco. Rev Paul Pediatr. 2013;31(2):172-81.

23. Bloch KV, Klein CH, Szklo M, Kuschnir MCC, Abreu GdA, Barufaldi LA, et al. ERICA: prevalences of hypertension and obesity in Brazilian adolescents. Rev Saúde Pública. 2016;50(Supl 1):1-12.

24. Rivera JA, Cossio TG, Pedraza LS, Aburto TC, Sanchez TG, Martorell R. Childhood and adolescent overweight and obesity in Latin America: a systematic review. Lancet Diabetes Endocrinol. 2014;2(4):32132. 
25. Farias ES, Santos AP, Farias-Júnior JC, Ferreira CRT, Carvalho WRG, Gonçalves EM, et al. Excess weight and associated factors in adolescents. Rev Nutr. 2012;25(2):229-36.

26. Gomes TN, Katzmarzyk PT, Santos FK, Souza M, Pereira S, Maia JA. Overweight and obesity in Portuguese children: prevalence and correlates. Int $\mathrm{J}$ Environ Res Public Health. 2014;11(11):11398-417.

27. Mihas C, Mariolis A, Manios Y, Naska A, Panagiotakos D, Arapaki A, et al. Overweight/obesity and factors associated with body mass index during adolescence: the VYRONAS study. Acta Paediatr. 2009;98(3):495500 .

28. Fortes LS, Conti MA, Almeida SS, Ferreira MEC. Body dissatisfaction in adolescents: a longitudinal study. Rev Psiquiatr Clín. 2013;40(5):167-71.

29. Cumming SP, Standage M, Gillison F, Malina RM. Sex differences in exercise behavior during adolescence: is biological maturation a confounding factor? J Adolesc Health. 2008;42(5):480-5.

30. Sherar LB, Esliger DW, Baxter-Jones AD, Tremblay MS. Age and gender differences in youth physical activity: does physical maturity matter? Med Sci Sports Exerc. 2007;39(5):830-5.

31. Allender S, Kremer P, Silva-Sanigorski A, Lacy K, Millar L, Mathews L, et al. Associations between activity-related behaviours and standardized BMI among Australian adolescents. J Sci Med Sport. 2011;14(6):512-21.

32. Hallal PC, Andersen LB, Bull FC, Guthold R, Haskell W, Ekelund U. Global physical activity levels: surveillance progress, pitfalls, and prospects. Lancet. 2012;380(9838):247-57.
33. Swinburn B, Shelly A. Effects of TV time and other sedentary pursuits. Int J Obes (Lond). 2008;32(Suppl 7):132-6.

34. Guerra PH, Farias JC Júnior, Florindo AA. Sedentary behavior in Brazilian children and adolescents: a systematic review. Rev Saúde Pública. 2016;50(9):115.

35. Pearson N, Biddle SJ. Sedentary behavior and dietary intake in children, adolescents, and adults. A systematic review. Am J Prev Med. 2011;41(2):178-88.

36. Ribeiro IC, Taddei JAA, Colugnatti F. Obesity among children attending elementary public schools in Sao Paulo, Brazil: a case-control study. Public Health Nutr. 2003;6(7):659-63.

37. Guimarães LV, Barros MBdA, Martins MSAS, Duarte EC. Fatores associados ao sobrepeso em escolares. Rev Nutr. 2006;19(1):5-17.

38. Pinto RP, Nunes AA, de Mello LM. Análise dos fatores associados ao excesso de peso em escolares. Rev Paul Pediatr. 2016;34(4):460-8.

39. Brevidelli MM, Coutinho RMC, Costa LFV, Costa LC. Prevalência e fatores associados ao sobrepeso e obesidade entre adolescentes de uma escola pública. Rev Bras Promoç Saúde. 2015;28(3):379-86.

\section{Endereço para correspondência:}

Eliane Denise Araújo Bacil

Rua Coração de Maria, 92

Bairro: Jardim Botânico

CEP: 80215370 - Curitiba - PR - Brasil

E-mail: elianebacil@hotmail.com 